ARTICLE

\title{
Olfactory expression of trace amine-associated receptors requires cooperative cis-acting enhancers
}

\author{
Ami Shah ${ }^{1,5}$, Madison Ratkowski ${ }^{1,5}$, Alessandro Rosa (i) ${ }^{2,3}$, Paul Feinstein ${ }^{2,3}$ \& Thomas Bozza (i) ${ }^{1,4 凶}$
}

Olfactory sensory neurons express a large family of odorant receptors (ORs) and a small family of trace amine-associated receptors (TAARs). While both families are subject to socalled singular expression (expression of one allele of one gene), the mechanisms underlying TAAR gene choice remain obscure. Here, we report the identification of two conserved sequence elements in the mouse TAAR cluster (T-elements) that are required for TAAR gene expression. We observed that cell-type-specific expression of a TAAR-derived transgene required either T-element. Moreover, deleting either element reduced or abolished expression of a subset of TAAR genes, while deleting both elements abolished olfactory expression of all TAARs in cis with the mutation. The T-elements exhibit several features of known OR enhancers but also contain highly conserved, unique sequence motifs. Our data demonstrate that TAAR gene expression requires two cooperative cis-acting enhancers and suggest that ORs and TAARs share similar mechanisms of singular expression.

\footnotetext{
${ }^{1}$ Department of Neurobiology, Northwestern University, Evanston, IL, USA. ${ }^{2}$ The Graduate Center Programs in Biochemistry, Biology and CUNY Neuroscience Collaborative, New York, NY, USA. ${ }^{3}$ Department of Biological Sciences, Hunter College, City University of New York, New York, NY, USA

${ }^{4}$ Chemistry of Life Processes Institute, Northwestern University, Evanston, IL, USA. ${ }^{5}$ These authors contributed equally: Ami Shah, Madison Ratkowski.

凶email: bozza@northwestern.edu
} 
vast majority of vertebrate genes are expressed from both the maternal and paternal alleles. However, a subset of genes is expressed from a single allele, a phenomenon referred to as monoallelic expression. For monoallelically expressed genes, the choice of expressed allele can be deterministic or random ${ }^{1,2}$. While monoallelic expression plays a central role in dynamic gene regulation, cell-type specification, phenotypic variability, and disease pathogenesis ${ }^{3}$, the mechanisms governing random monoallelic gene choice are ill-defined.

The mammalian olfactory system provides an intriguing example of random monoallelic expression. Odor detection is mediated by a large repertoire of olfactory receptor genes-over 1000 genes in mice. Each mouse olfactory sensory neuron (OSN) in the nasal cavity chooses to express one allele of one olfactory receptor gene from the repertoire of over 2000 alleles-so-called singular expression ${ }^{4}$. Olfactory receptors come in two phylogenetically distinct clades: odorant receptors (ORs) and Trace Amine-Associated Receptors (TAARs), both of which are subject to singular expression ${ }^{5,6}$.

The mechanisms of singular expression have been studied most extensively using mouse ORs as a model. The ORs come in two phylogenetically distinct families ${ }^{7-10}$. The class I OR family comprises roughly 130 intact genes located in a single large gene cluster, while the class II family comprises over 900 intact genes, most of which are located in over 50 clusters scattered throughout the genome ${ }^{11}$. The expression of some OR genes depends on short, proximal regulatory sequences ${ }^{12,13}$, while other genes require long-range, cis-acting enhancers ${ }^{14}$. The first identified enhancer, the $\mathrm{H}$ element, is located approximately 55 kilobases (kb) upstream of the MOR28 (Olfr1507) gene cluster ${ }^{14,15}$. Since the discovery of $\mathrm{H}$, scores of similar enhancers have been identified scattered in and around $\mathrm{OR}$ gene clusters ${ }^{16-21}$. These include the $\mathrm{P}$ element in the $\mathrm{P} 2$ (Olfr17) cluster, the $\mathrm{A} / \mathrm{J}$-core element in the class I OR cluster 20,22 and the large set of Greek Islands ${ }^{19,21}$.

These long-range enhancers and some proximal promoters contain conserved DNA sequence motifs, such as Olf1/Ebf $(\mathrm{O} /$ E)-like and homeodomain binding (HD) sites that have been implicated in olfactory-specific gene choice ${ }^{17,23-26}$. In addition, OR gene choice is partly dependent on the LIM homeodomain protein transcription factor Lhx2, which binds to the $\mathrm{HD}$ sites of OR promoters and enhancers ${ }^{27-29}$. Interestingly, the Greek Islands are enriched in co-bound $\mathrm{O} / \mathrm{E}$ and $\mathrm{HD}$ sites $^{21}$. The $\mathrm{H}$ (core), $\mathrm{P}$, and A (J core) enhancers contain multiple copies of a characteristic HD site (TAATGA), including an extended HD sequence, CTTTTTAATGA ${ }^{17,30}$. Two of the $\mathrm{HD}$ sites in the $\mathrm{H}$ element are required for its enhancer activity ${ }^{31}$. Moreover, inserting multiple tandem copies of the HD site into an OR transgene dramatically increases probability of expression ${ }^{17,30,32}$. Thus, HD sites are a critical determinant for OR choice.

Beyond these sequence motifs, epigenetic mechanisms have been proposed to play a central role in OR gene regulation. The available evidence suggests that singular expression involves a complex interaction between enhancers and proximal promoters to selectively release a single OR allele from epigenetic repression ${ }^{19,21,33-35}$. OR gene clusters are marked as constitutive heterochromatin by specific histone modifications (H4K20me3 and $\mathrm{H} 3 \mathrm{~K} 9 \mathrm{me} 3$ ) which silence gene transcription ${ }^{33}$. This repression correlates with a change in nuclear localization of OR loci from the nuclear lamina to internal nuclear aggregates ${ }^{36}$. Conversely, expression of a given OR locus is correlated with a change from repressive to permissive $\mathrm{H} 3 \mathrm{~K} 4 \mathrm{me} 3$ histone marks, indicating a change in histone state correlated with singular expression $^{33}$. Thus, epigenetic repression and specific nuclear localization may be central to the mechanism of OR gene choice.
By contrast, little is known about the mechanism of TAAR gene choice. The TAAR family in mouse comprises 15 genes, all of which are located in a single genomic cluster with no intervening genes ${ }^{37}$. Like ORs, all of the TAARs (except Taar1) are subject to singular expression in subsets of OSNs ${ }^{6,37-39}$. Unlike ORs, TAARs exhibit some differences that are not easily reconciled with features of OR gene choice. First, the TAAR gene cluster lacks the enrichment in epigenetic marks that are thought to silence class II OR gene clusters, at least when looking across all mature OSNs ${ }^{40}$. Second, it has been suggested that TAARdeletion alleles behave differently from OR-deletion alleles-that OSNs expressing an OR deletion allele switch to express an alternate gene and silence the initially chosen locus, while TAAR deletion alleles appear to remain active ${ }^{40}$. Third, while most class I/II OR genes are located in several internal heterochromatic centers in the nucleus, the TAAR genes are located preferentially at the nuclear lamina prior to choice ${ }^{36,41}$. In fact, it has been suggested that the mechanism of TAAR gene expression is fundamentally different from that of ORs ${ }^{40}$. However, the genetic mechanisms underlying TAAR gene regulation are poorly characterized, and no enhancer or promoter sequences have been identified that are necessary to promote TAAR gene expression.

Here, we identify two phylogenetically conserved enhancers in the TAAR gene cluster and show that they are necessary and sufficient to promote TAAR gene expression. The TAAR enhancers exhibit both unique and shared features with known OR enhancers. Our findings establish a powerful model for studying olfactory receptor gene choice and suggest that TAAR and OR gene expression take place via a similar basic mechanism.

\section{Results}

TAAR4 transgene expression requires an enhancer. To begin defining the minimal promoter sequences required for TAAR gene expression, we generated a TAAR4 transgene consisting of a genomic fragment encompassing $\sim 2.3 \mathrm{~kb}$ upstream of the Taar4 transcription start site (determined by $5^{\prime} \mathrm{RACE}$ ), the single intron, and the endogenous polyadenylation site (Fig. 1a). The transgene was modified so that the Taar4 coding sequence was deleted and replaced with that of YFP. The resulting transgene ( $\triangle \mathrm{T} 4$-YFPtg) failed to be expressed in 5 independent founder lines (Fig. 1b, c). This suggested that the included putative promoter was insufficient to impart gene choice, or that the transgene was otherwise non-functional.

To test the functionality of the transgene, we appended a strong OR enhancer ( $5 \mathrm{x}$ repeat of an extended homeodomain binding site), $5 \times 21 \mathrm{HD}^{30}$, to the $5^{\prime}$ end of the $\Delta \mathrm{T} 4$-YFPtg (Fig. 1d). The resulting transgene $(5 \mathrm{xHD}-\Delta \mathrm{T} 4$-YFPtg) was robustly expressed in OSNs of the olfactory epithelium in 5 out of 5 independent lines (Fig. 1e). All but one of the transgenic lines showed a characteristic pattern of expression, with robust labeling of OSNs of the dorsal main olfactory epithelium and in VSNs of the vomeronasal organ (Fig. 1e; Fig. S1). They also shared a common pattern of glomerular projections to the dorsolateral olfactory bulb and accessory olfactory bulb (Fig. If and Fig. S1). This indicated that the $\Delta \mathrm{T} 4$-YFPtg transgene is functional for gene expression when an olfactory enhancer sequence is provided.

Identification of putative TAAR enhancers. By analogy to canonical ORs, we reasoned that TAAR gene expression may require cis-acting enhancers that are located in the cluster but that are missing from the TAAR4 transgene. To identify putative enhancers, we searched the mouse TAAR cluster between the conserved genes $V n n 1$ and Stx7 for sequences that were: (1) conserved across eutherian mammals, (2) non-repetitive, and 
a
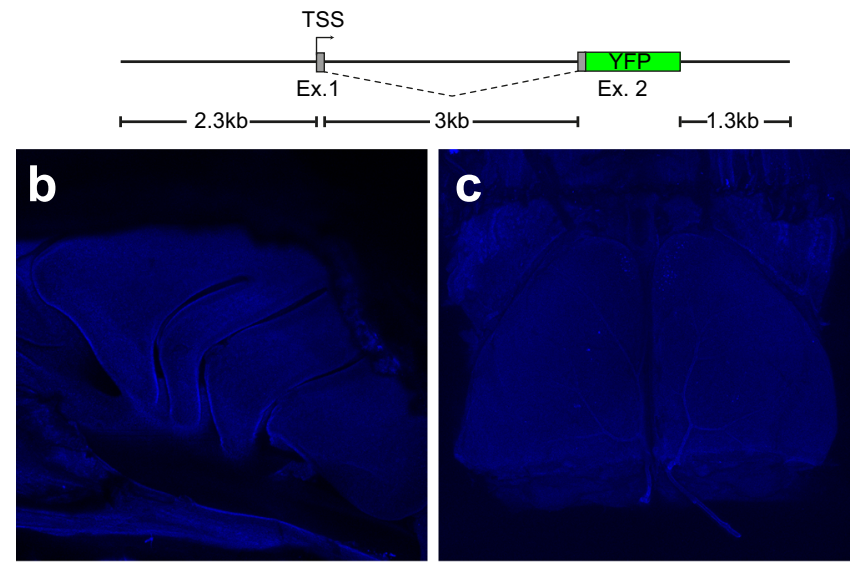

d

$5 \times \mathrm{HD}$
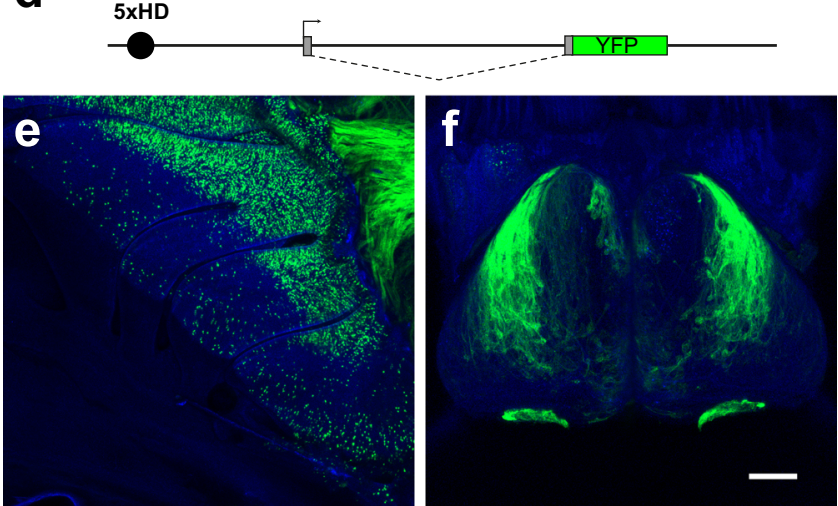

Fig. 1 TAAR4 transgene expression requires a strong OR enhancer. $A$ transgene derived from the endogenous Taar4 locus is not sufficient to drive reporter expression. a Schematic of the transgene backbone in which the Taar4 coding sequence in exon 2 (Ex. 2) is replaced with that of YFP (green box). Non-coding regions shown in gray. Defined transcription start site (TSS) is indicated (arrow). b Confocal image showing a wholemount of the olfactory turbinates of a $\Delta$ T4-YFPtg mouse showing lack of labeled OSNs. c Confocal image showing a wholemount of the dorsal olfactory bulb showing no labeled axons. d Diagram of the $5 \times \mathrm{HD}-\Delta \mathrm{T} 4-\mathrm{YFPtg}$, in which a strong OR enhancer ( $5 \times \mathrm{HD}$; black circle) is placed upstream of the putative promoter. e Wholemount fluorescence image of the olfactory turbinates showing robust expression of YFP in OSNs primarily in the dorsal olfactory epithelium. $\mathbf{f}$ YFP + axons of OSNs primarily innervate the dorsolateral olfactory bulb and accessory olfactory bulb. Scale bar in $\mathbf{f}=500 \mu \mathrm{m}$ in $\mathbf{b}, \mathbf{c}$, $\mathbf{e}$, and $\mathbf{f}$.

(3) intergenic (i.e., outside known coding sequences, UTRs, or pseudogene fragments; Fig. 2a). To search for conservation, we used a combination of basewise sequence comparison (percent identity plots; PipMaker ${ }^{42}$ between mouse and human, and the phyloP conservation track for 40 eutherian mammals in the UCSC database) (Fig. 2b). We identified two conserved intergenic regions that matched our criteria-one between Taar1 and Taar2 and one between Taar6 and Taar7a (Fig. 2b). These sequences were named T-elements 1 and 2 (TE1 and TE2), respectively.

The T-elements share some features with OR enhancers. Known OR enhancers contain $\mathrm{HD}$ and $\mathrm{O} / \mathrm{E}$-like binding sites $^{17,20,27}$. We therefore searched for putative HD and O/E-like sites in TE1 and TE2 and looked for conservation that might suggest function. Each element contains seven instances of the core HD binding motif TAATNN. In TE1, four of these copies are conserved including one instance each of TAATAG,
TAATGA, TAATTA, and TAATCA (Fig. 3a, c and S2). Similarly, four HDs are conserved in TE2, one instance of TAATCC and a triad of TAATGA sequences (Fig. 3a, c and S2). This triple repeat of TAATGA located within $206 \mathrm{bp}$ of each other (a motif that is known to influence choice ${ }^{17,30}$ ) is only found once in the TAAR cluster (in TE2). Similarly, there are conserved HDs in 6 known functional OR enhancers: H-core, P, A (J core) and the Greek Islands Lipsi, Sfaktiria and Kefallonia (Fig. 3b, c and S3). Thus, as with known OR enhancers, the T-elements contain multiple copies of conserved HD binding sites.

Next, we searched TE1 and TE2 for O/E-like sites that closely match the degenerate consensus sequence YCCCNNGGGR ${ }^{21,43-45}$. We identified three such sites in both mouse TE1 and TE2; however, they were not conserved across species. In contrast, we could easily locate well-conserved O/Elike sites in all of the OR enhancers except $\mathrm{H}$ (Fig. S4), which contains an $\mathrm{O} / \mathrm{E}$-like site only in mouse. Thus, unlike OR enhancers, TE1 and TE2 appear to lack recognizable, conserved $\mathrm{O} / \mathrm{E}$-like sites.

The Greek Islands are defined in part by chromatin accessibility peaks and binding of transcription factors Lhx2, Ldb1, and Ebf ${ }^{21}$. To examine whether the T-elements share such features with the 6 known functional OR enhancers, we analyzed published ATAC-seq and ChIP-seq data from mature (OMPexpressing) OSNs ${ }^{21,27}$. In this data set, both TE1 and TE2 show similar accessibility to Kefallonia, A (J core) and H (Fig. 4). However, neither TE1 nor TE2 was as enriched for binding of Lhx2, Ebf1, or Ldb1 as most of the known OR enhancers. This lack of binding was also observed for the $\mathrm{P}$ element. Thus, averaged across all OMP-expressing OSNs, TE1 and TE2 exhibit some, but not all, features of known OR enhancers. It should be noted that TAAR OSNs represent a small fraction of the total OSN pool and some of these enrichments of binding may only be observed in TAAR-expressing OSNs (see Discussion).

The T-elements contain a unique, shared motif. Our analysis also revealed a $\sim 30 \mathrm{bp}$ block of conserved sequence that is common between the two T-elements (Fig. 3a, d). This motif, referred to as Shared Homology in the T-Elements (SHiTE), contains two tandem conserved sequences, TTGCATCA and TAAAGTTTTC. We searched for the SHiTE motif against a database of known OR enhancers including the 63 Greek Islands using FIMO (Meme suite $^{46}$ ) and BLAST. No significant matches were found (FDR $q<$ $0.05)$. The only close match $(q=0.086)$ was in the Greek Island Evia and consisted of a stretch of homology encompassing the SHiTE motif, AAAGTTTTCT. In a genome wide search, we found no matches to the full-length SHiTE motif outside of the TAAR cluster. While matches to shorter stretches of SHiTE (often including AAAGTTT) are found throughout the genome, the full SHiTE motif seems to be unique to the TAAR elements.

The T-elements drive transgene expression. To test whether the T-elements function as enhancers for TAAR genes, we appended TE1 (698 bp) or TE2 (345 bp) to the $5^{\prime}$ end of the $\Delta$ T4-YFPtg to see if each element could rescue transgene expression (Fig. 5a). Interestingly, addition of either TE1 or TE2 drove robust transgene expression in OSNs (Fig. 5b, d). TE1 (TE1- $\Delta$ T4-YFPtg) drove strong expression in 2 of 3 independent transgenic lines, with sparsely labeled neurons located throughout the olfactory epithelium (Fig. 5b). TE2 (TE2- $\Delta$ T4-YFPtg) drove strong expression in 4 of 5 lines, with one line showing the highest numbers of OSNs in the dorsal zone of the epithelium (Fig. 5d), and others showing significant expression in more ventral regions, and in the septal organ (Fig. S1). (Because TAAR expression has not been reported in the septal organ, this likely 

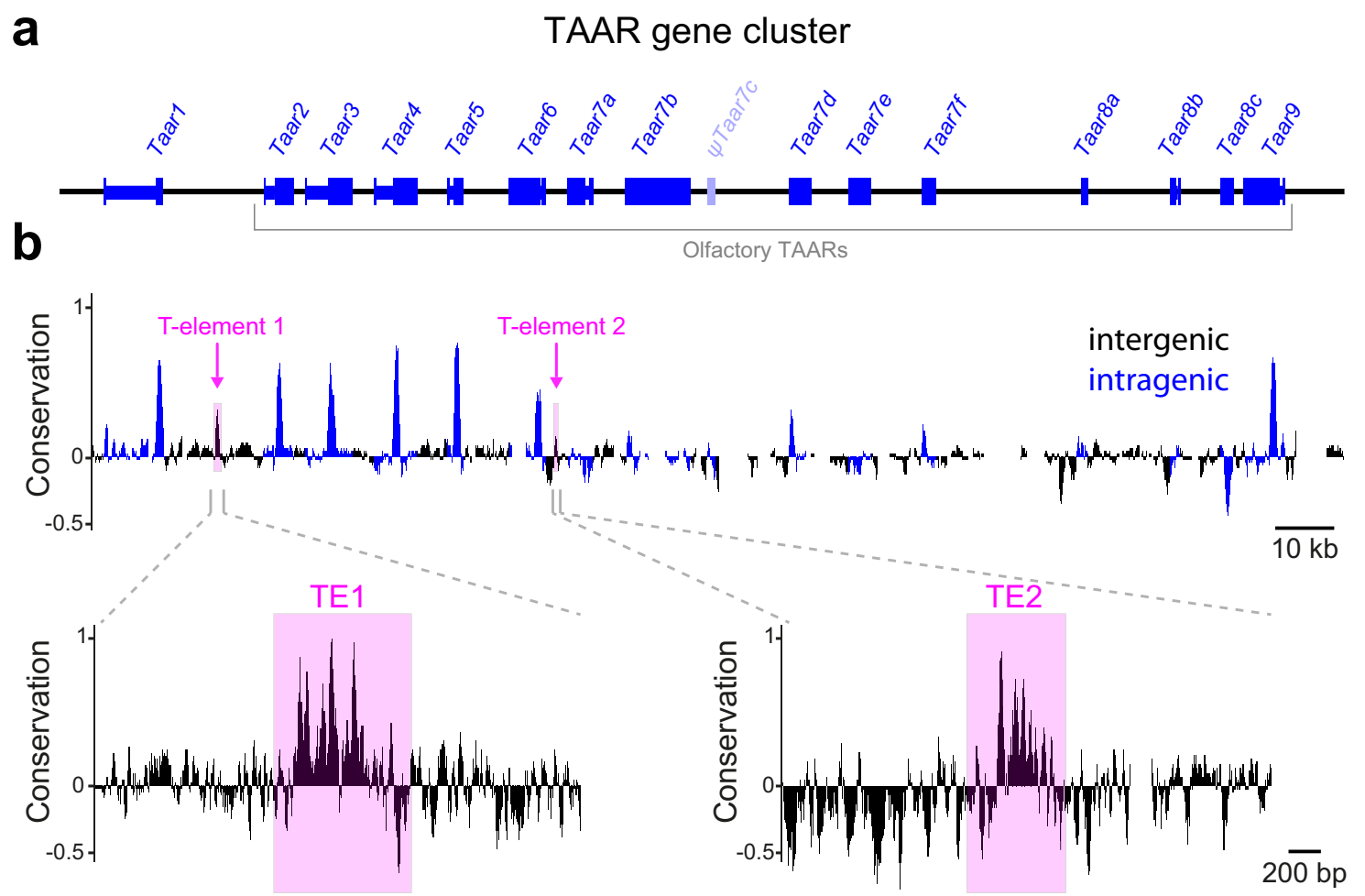

Fig. 2 Identification of putative enhancers in the TAAR cluster. a Gene structure of all 15 intact TAARs (blue) and the pseudogene $\psi$ Taar7c (light blue). Exons (thick lines) and introns (thin lines) are indicated. b Basewise conservation across eutherian mammals in the TAAR cluster determined using PhyloP. Regions within known genes are shown in blue, intergenic regions in black. Two intergenic regions that have high conservation peaks and HD sequences (TE1 and TE2) are highlighted (magenta). Conservation track is expanded below.

represents ectopic expression.) Thus, like the $5 \times 21 \mathrm{HD}$ enhancer, both TE1 and TE2 can rescue the expression of the $\Delta$ T4-YFPtg, indicating that they have enhancer activity.

The T-elements drive expression in TAAR sensory neurons. Because the Taar4-derived transgene lacks a receptor coding sequence, YFP expressing OSNs would be expected to express an alternate receptor gene, which would then direct axons to specific glomeruli in the olfactory bulb ${ }^{14,47,48}$. Consequently, the pattern of innervation in the bulb provides information about which cell types express the transgene $\mathrm{e}^{6,16,49}$.

We noted that, in specific T-element transgenic lines, YFPlabeled axons converged to multiple glomeruli in the dorsomedial olfactory bulb (Fig. 5c, e) in a pattern that resembled the distribution of dorsal TAAR glomeruli6,40,41. This pattern of innervation was not seen in any of the $5 \mathrm{xHD}-\Delta \mathrm{T} 4$-YFPtg lines, which tend to label glomeruli in the dorsal-lateral bulb (Fig. 1f) in a DII (dorsal class II) pattern ${ }^{16}$. To determine if T-element transgene-expressing OSNs were preferentially innervating TAAR glomeruli, we crossed one line for each transgene to the genetargeted mouse strain, $\triangle \mathrm{T} 4-\mathrm{RFP}$, in which the coding sequence for TAAR4 is replaced with that of a red fluorescent marker, thereby labeling all dorsal TAAR glomeruli ${ }^{6}$. Wholemount and histological analysis showed that all RFP-labeled glomeruli were targeted by YFP-labeled axons (Fig. $5 \mathrm{f}-\mathrm{i}$ ). While the relative contribution of $\Delta \mathrm{T} 4$ and transgenic axons (i.e., the fraction of red to green axons) varied across glomeruli, the preferential innervation of TAAR glomeruli indicated that transgeneexpressing OSNs preferentially express TAARs.

To directly test for selective TAAR expression in transgenelabeled OSNs, we performed combined immunohistochemistry for YFP protein and in situ hybridization using pooled probes for 6 representative class I ORs, class II ORs, and TAARs (Fig. 5j, l).
If expression of intact receptor genes were random across all receptor types, each probe pool should exhibit a similar, low level of co-expression. Contrary to this prediction, the co-expression rate observed with the TAAR-probe pool was significantly higher than that for the class I and class II pools. This was observed for both transgenes (Fig. 5k, m). Thus, for the lines analyzed, transgene-expressing OSNs exhibited a bias towards expressing TAAR genes. Taken together, the data indicate that the Telements may influence the cell-type-specific expression of TAAR genes.

The T-elements are required for TAAR gene expression. To test whether TE1 and TE2 are necessary for TAAR gene expression, we generated deletions of each element alone, or together in cis, using CRISPR/Cas9-mediated gene editing. Guide RNAs were designed to PAM sequences flanking each of the elements (Fig. 6a). Gene editing was carried out in mice harboring modified alleles of Taar1 (Taar1-YFP) and Taar4 (Taar4-RFP), allowing us to track the expression of these genes in cis with the mutations. We isolated three mutations: (1) a double cis deletion of TE1 and TE2 $(\triangle \mathrm{TE} 1 / 2)$ linked to Taar4-RFP (Fig. 6a); (2) a deletion of TE1 ( $\triangle \mathrm{TE} 1)$ linked to Taar1-YFP; and (3) a deletion of TE2 ( $\triangle \mathrm{TE} 2)$ linked to Taar1-YFP (see below).

We first analyzed $\Delta \mathrm{TE} 1 / 2$ mice, in which both elements were deleted on the same chromosome in cis with the Taar4-RFP allele (Fig. 6a). Taar4-RFP (with intact elements) normally produces robust labeling of OSNs in the dorsal epithelium ${ }^{6}$ (Fig. 7). Notably, in $\triangle \mathrm{TE} 1 / 2$ homozygous mice, no RFP expression was seen from the linked Taar4-RFP allele (Fig. 7). To define the extent of the effect of the double deletion, we measured expression of all TAARs in the olfactory mucosa of homozygous $\Delta \mathrm{TE} 1 / 2$ mice via qPCR. In contrast to wild-type littermates, homozygous $\Delta \mathrm{TE} 1 / 2$ mice showed no expression of TAAR genes, 

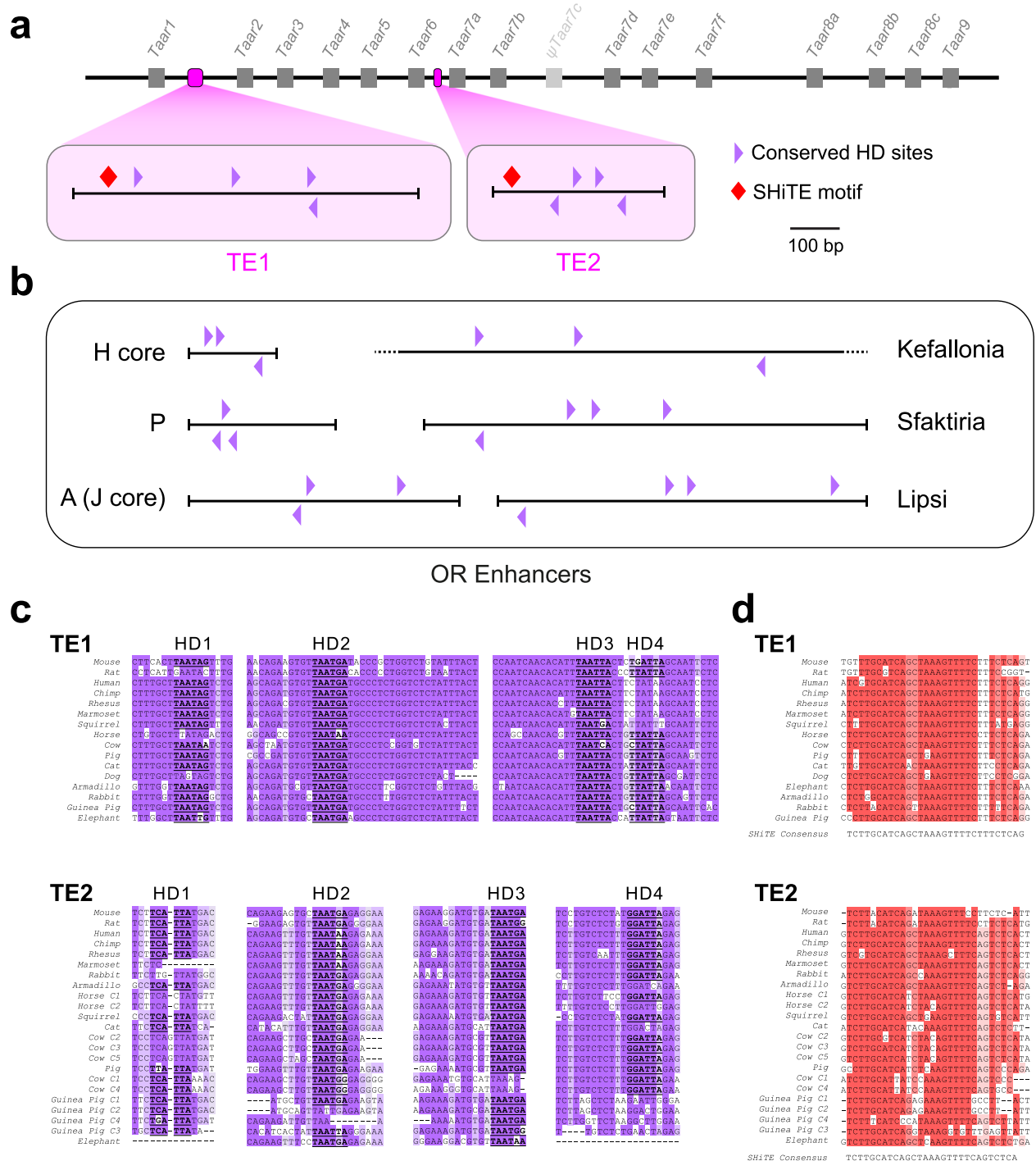

Conserved Homeodomain Sites

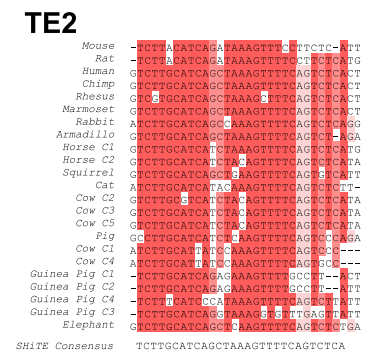

SHITE Motif

Fig. 3 The T-elements comprise conserved homeodomain binding sites and a novel shared motif. a Schematic of the TAAR cluster showing relative positions of TE1 and TE2 (magenta). Genes are indicated by gray boxes. Schematic of the T-elements (bottom) showing placement of phylogenetically conserved HD sites (purple triangles). The T-elements also contain a highly conserved, shared homology, the SHiTE motif (red diamonds). b Schematic of known functional OR enhancers showing positions of conserved HD sites. c Alignments of segments of TE1 and TE2 from 16 mammalian species showing all conserved HD sites, HD1-4 in each. d Alignment of the TE1 and TE2 shared homology (SHiTE) motifs across representative mammalian species. Single alignment is split, top (TE1) and bottom (TE2).

while expression of a set of control OR genes was unaffected (Fig. 6b, S5). This indicates that TE1 and/or TE2 are necessary for expression of TAARs in the olfactory epithelium.

To determine whether the effects of deleting the elements was TAAR-specific, we performed RNA-seq on olfactory mucosa from homozygous $\Delta \mathrm{TE} 1 / 2$ and wild-type littermates. The element deletion did not affect expression of genes that immediately flank the TAAR cluster and that are expressed in the olfactory mucosa -Slc18b1, Vnn1 and Vnn3, Stx7 and Moxd1 (Fig. 6c). In addition, the only olfactory receptors that exhibited significantly reduced expression in $\triangle \mathrm{TE} 1 / 2$ mice were TAARs (Fig. 6d). Across all genes expressed in the olfactory mucosa, only one non-TAAR gene (E030030I06Rik) was significantly downregulated (Fig. 6e). Therefore, reduced expression caused by the $\Delta \mathrm{TE} 1 / 2$ mutation is specific to the TAAR gene cluster.

To determine if TE1 and TE2 act exclusively in cis (on the same allele), we looked for RFP expression in heterozygous $\Delta \mathrm{TE} 1 / 2$ mice in which the elements are present in trans, but lacking in cis, of the Taar4-RFP allele (Fig. 7a). We observed no RFP expression from the Taar4-RFP allele in heterozygous $\Delta \mathrm{TE} 1 / 2$ mice (Fig. $7 \mathrm{~b}$, c), indicating that the elements function solely in cis.

Thus, knocking out both TE1 and TE2 in cis completely and selectively abolishes expression of the linked TAAR genes in the olfactory epithelium.

The T-elements have a combinatorial effect on TAAR gene expression. To define the specific contribution of TE1 and TE2 to TAAR gene expression, we quantified gene expression in the olfactory mucosa of single element deletion mice. Both the $\Delta \mathrm{TE} 1$ and $\triangle \mathrm{TE} 2$ mutations were generated independently in cis with a Taar1-YFP allele (Fig. 8a), allowing us to observe whether deleting either element induced Taarl gene expression in the epithelium (i.e., normally insulate Taar1 from being chosen). However, we did not observe Taar1-YFP expression in the olfactory epithelium of either strain (not shown). 


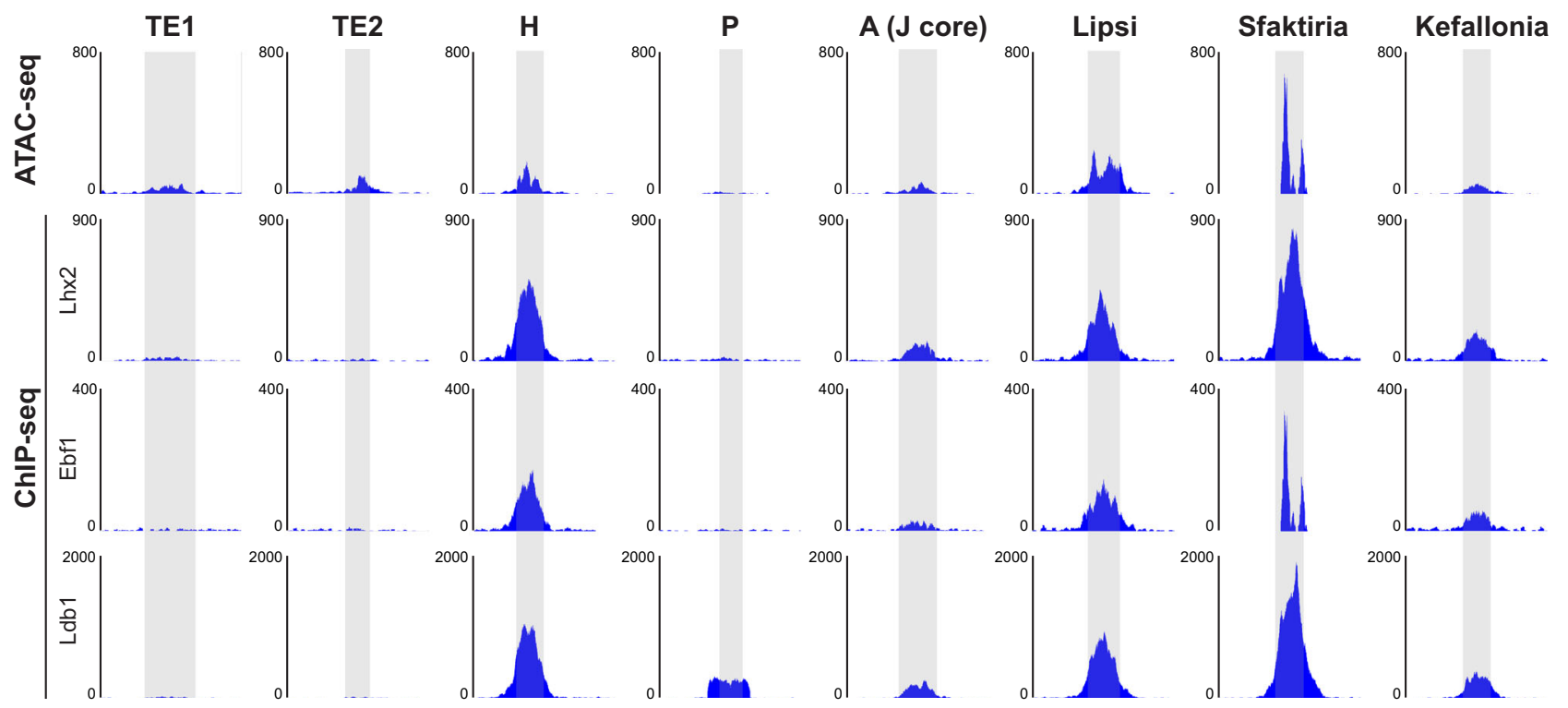

Fig. 4 TE1 and TE2 share some but not all epigenetic features of OR enhancers. ATAC-seq and ChIP-seq data from all mature OSNs ${ }^{21,27}$ in genomic regions corresponding to TE1 and TE2, and known functional OR enhancers: H, P, A (J core), Lipsi, Sfaktiria and Kefallonia. T-elements show similar chromatin accessibility, but lack Lhx2, Ebf1 (O/E), and Ldb1 binding that is observed in most of the known functional OR enhancers.

Next, we quantified olfactory TAAR expression via qPCR. $\triangle T E 1$ mice showed dramatically reduced expression of all TAAR genes except Taar7e and Taar7f, with the affected TAARs being essentially undetectable (Fig. 8b, S5). It is interesting to note that the effect of this deletion was not restricted to adjacent TAARs (e.g., Taar2 and Taar3), but extended to the opposite end of the cluster (e.g., Taar9). Thus, TE1 has a strong influence on expression of almost all olfactory TAARs. On the other hand, $\triangle T E 2$ mice showed a significant reduction in expression of a different constellation of TAAR genes including Taar3, 4, 5, 6, 7a, $8 a / b / c$, and 9 (Fig. $8 c$, S5). Several TAAR genes (Taar $2,7 b, 7 d, 7 e$, and $7 f$ ) were relatively spared. Overall, the effect of the TE2 deletion was less pronounced than for TE1. The effects of the two deletions were distinct but overlapping-some genes (e.g., Taar3) were severely downregulated by both mutations, while Taar7e was generally spared by both. Interestingly, there was no clear relationship between the zone of expression and dependence on either element. For example, deletion of TE1 reduced expression of both dorsal (e.g., Taar2 and Taar9) and ventral (e.g., Taar7a and Taar7b) genes. Thus, each element has differential and overlapping effects on TAAR gene expression.

Taken together, our data indicate that TE1 and TE2 are cisacting enhancers that function in a combinatorial fashion to selectively promote olfactory expression of genes in the TAAR cluster.

\section{Discussion}

Most of what is known about the mechanisms of olfactory singular expression has come from studying ORs. Consequently, the relationship between mechanisms of TAAR and OR gene choice is not well understood. Here, we identify two conserved enhancers in the TAAR gene cluster (TE1 and TE2) and show that deletion of both abolishes TAAR gene expression in OSNs. We further show that the enhancers function cooperatively, with each affecting an overlapping subset of TAAR genes. A recent study has identified similar regulatory sequences and corroborates these findings ${ }^{50}$. Interestingly, the $\mathrm{T}$-elements share features with known OR enhancers (i.e., the presence of conserved HD binding motifs), but also contain conserved, common motifs that appear specific to the T-elements and that may underlie critical aspects of their function. Overall, our data indicate that TAAR and OR gene regulation share a common basic mechanism. Furthermore, the TAAR cluster is the only olfactory receptor gene cluster for which all the genetic elements that are required for expression are known. Therefore, the TAAR cluster provides a powerful model to explore mechanisms of olfactory receptor gene choice.

Previous studies have deleted single OR enhancers in a given gene cluster, and have shown distance-dependent, partial suppression of OR gene expression ${ }^{15,18-20,22}$. Deleting the enhancers $\mathrm{H}, \mathrm{P}$, and Lipsi in class II OR clusters impacts genes over a distance of $\sim 200 \mathrm{~kb}^{15,18,19}$, while deleting A (J core) in the class I OR cluster affects genes as far away as $3 \mathrm{Mb}^{20,22}$. In all cases, expression persists for many genes in the clusters. This has led to the idea that multiple enhancers are required to drive expression of all genes in a given cluster. While likely, this has not been shown directly and it is difficult to exclude the possibility that some genes in the cluster are simply independent of long-range enhancers. The effects of TE1 and TE2 provide an example of cooperative enhancer function that is required for expression of all genes in an olfactory cluster.

We show that, while the T-elements function together to regulate all olfactory TAAR genes, each element alone influences expression of distinct, overlapping sets of genes. The effect of single T-element deletions was not obviously correlated with distance from the enhancers-something that was also seen with deletion of the $\mathrm{P}$ element ${ }^{18}$. We note that the TAAR cluster is relatively small $(\sim 200 \mathrm{~kb})$ compared with typical OR gene clusters (mean size $\sim 1.2 \mathrm{Mb}^{7}$ ), which means that TE1 and TE2 could affect the entire TAAR cluster even if their range is similar to that of the OR enhancers.

Our previous data show that TAAR genes residing on the distal ends of the cluster are expressed in the dorsal zone of the olfactory epithelium, while those in the middle of the cluster (near TE2) are expressed ventrally or broadly across zones ${ }^{6}$. This might indicate that TE2 influences zonal expression and/or selectively drives expression of the interior genes. Interestingly, we did not observe a clear relationship between zone of expression and susceptibility to deletion of TE1 or TE2-both single element deletions affected dorsally and ventrally expressed TAARs. Additionally, our TE2-containing TAAR4 transgene 

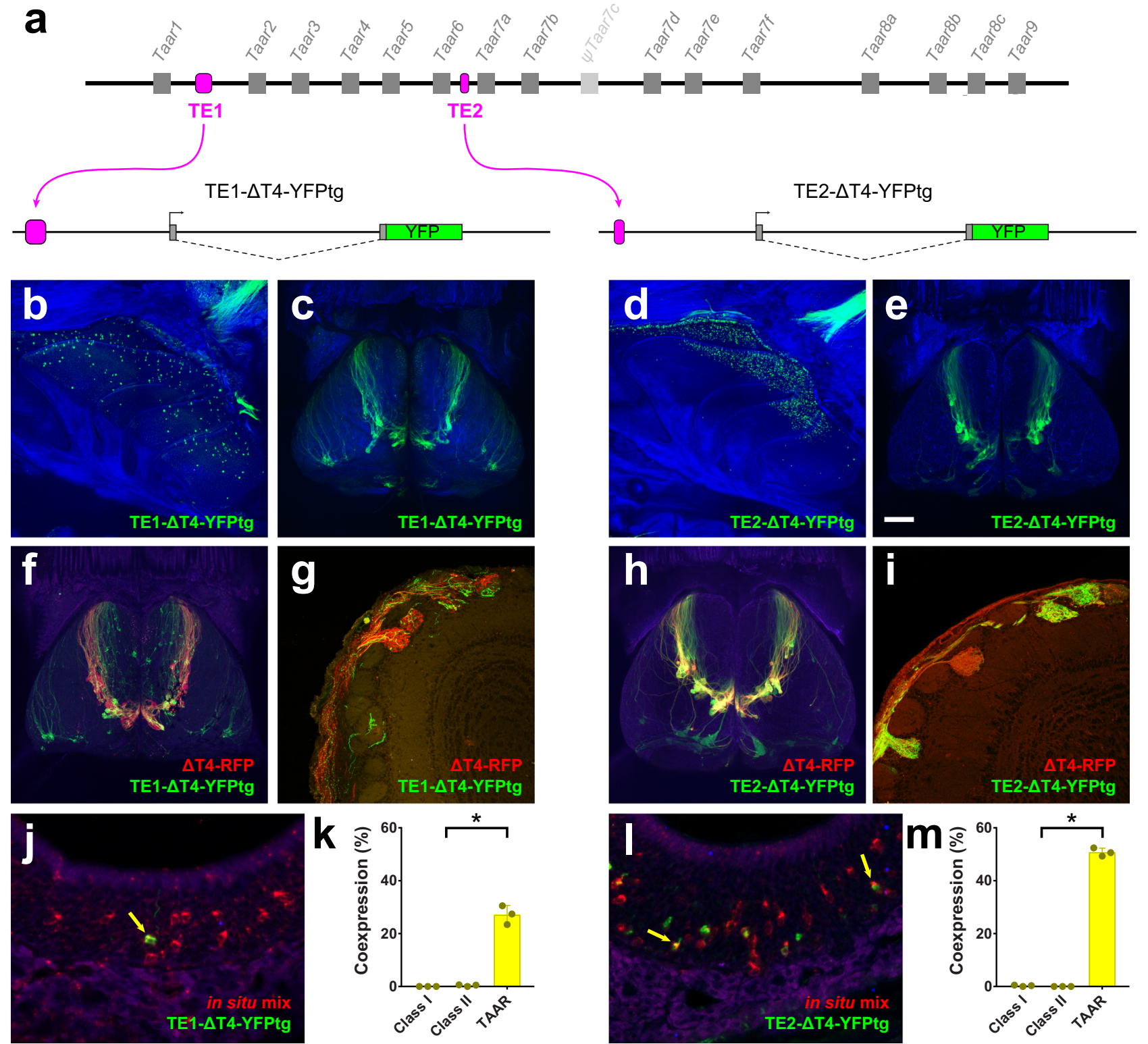

Fig. 5 TE1 and TE2 drive expression of the TAAR4 transgene. a Top, schematic of TAAR cluster showing placement of TE1 or TE2 (magenta). Bottom, diagram of $\triangle$ T4-YFP transgenes in which TE1 and TE2 are inserted upstream of the Taar4 promoter and YFP coding sequence (green). b-e Wholemount confocal images of olfactory epithelium and olfactory bulb of TE1- $\Delta$ T4-YFPtg and TE2- $\Delta$ T4-YFPtg mice showing YFP expression in OSNs in the epithelium and labeled axons and glomeruli in the dorsal olfactory bulb. OSN axons project to a cluster of dorsal glomeruli, reminiscent of the endogenous TAAR glomeruli. $\mathbf{f}$ Confocal image of dorsal olfactory bulbs, and $\mathbf{g}$ histological section, of a mouse that has the TE1- $\Delta$ T4-YFPtg and a $\Delta$ T4-RFP targeted allele. RFP + and YFP+ axons target the same glomeruli. $\mathbf{h}$ Confocal image of dorsal olfactory bulbs, and $\mathbf{i}$ histological section, of a mouse that has the TE2- $\Delta T 4-$ YFPtg and a $\triangle$ T4-RFP targeted allele. RFP+ and YFP+ axons target the same glomeruli. $\mathbf{j}$, I Combined in situ hybridization (TAAR probe mix, red) and immunohistochemistry for YFP (green) in histological sections of olfactory epithelium from TE1- $\Delta$ T4-YFPtg (j) and TE2- $\Delta$ T4-YFPtg (I) mice. Coexpressing cells indicated by yellow arrows. $\mathbf{k}, \mathbf{m}$ Fraction of YFP+ cells that label with the class I, class II, or TAAR probe pools in TE1- $\Delta T 4-Y F P t g$ (k) and TE2- $\Delta$ T4-YFPtg $(\mathbf{m})$ animals $\left(n=3\right.$ mice). Error bars indicate mean \pm SEM. ${ }^{\star} p<0.0001$; two-sided, one-way ANOVA, Dunn-Šidák correction for multiple comparisons. Scale bar in $\mathbf{e}=500 \mu \mathrm{m}$ in $\mathbf{b}, \mathbf{c}, \mathbf{d}, \mathbf{e}, \mathbf{f}$, and $\mathbf{h} ; 100 \mu \mathrm{m}$ in $\mathbf{g}$ and $\mathbf{i} ; 25 \mu \mathrm{m}$ in $\mathbf{j}$ and $\mathbf{I}$.

reliably drove expression in the dorsal epithelium, while the TE1 transgene tended to drive expression more broadly. Thus, the transgene expression patterns do not support a simple model in which TE2 is the sole director of ventral/broad expression, as suggested by its position among the ventrally/broadly expressed genes. Future studies examining promoter/enhancer interactions may shed light on what determines zonal expression of TAAR genes.

Most putative OR enhancers (i.e., the Greek Islands) have been defined operationally based on accessibility and transcription factor binding ${ }^{19,21}$. A handful of enhancers have been functionally defined by adding the sequences to transgenes or by deleting the sequences in mice ${ }^{15,17-20,22}$. It is worth noting that the lack of complete silencing of OR gene expression in all previous enhancer deletions leaves open questions about the actual minimal sequences that are required for enhancer function. Transgenic expression reports how a specific enhancer fragment can function to promote expression but does not confirm that the entire enhancer is present on the transgene. Similarly, the partial knockdown of OR gene expression seen for single enhancer 
a

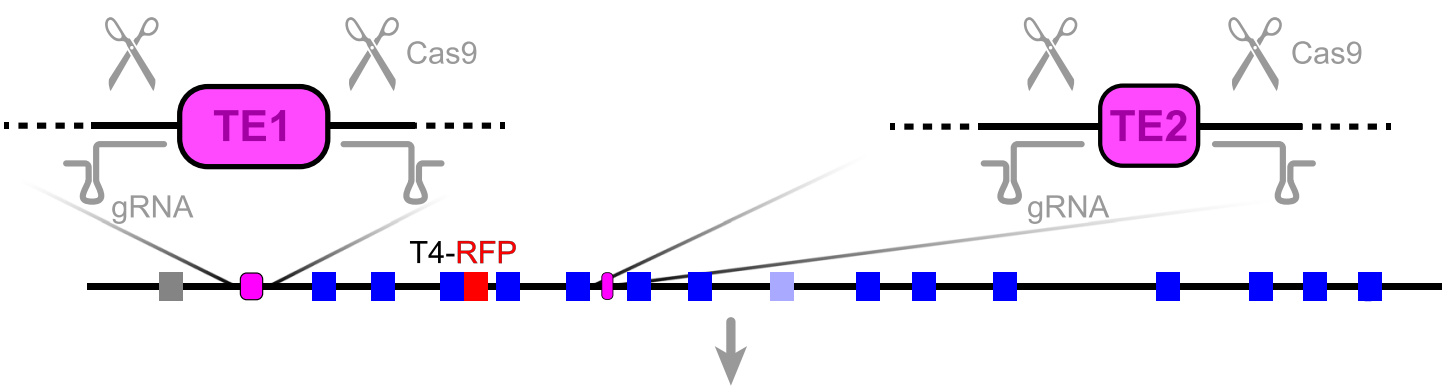

$\Delta \mathrm{TE} 1 / 2$

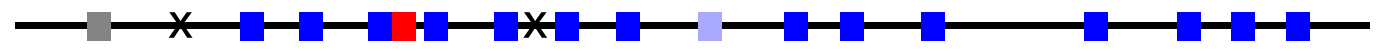

b

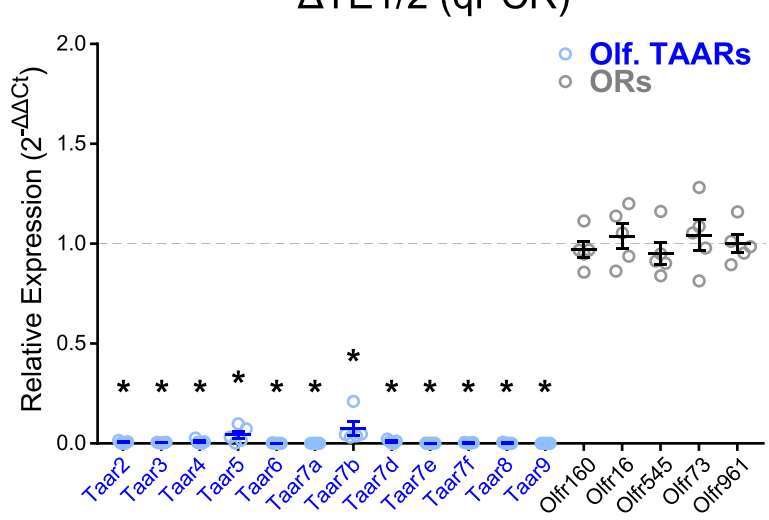

d

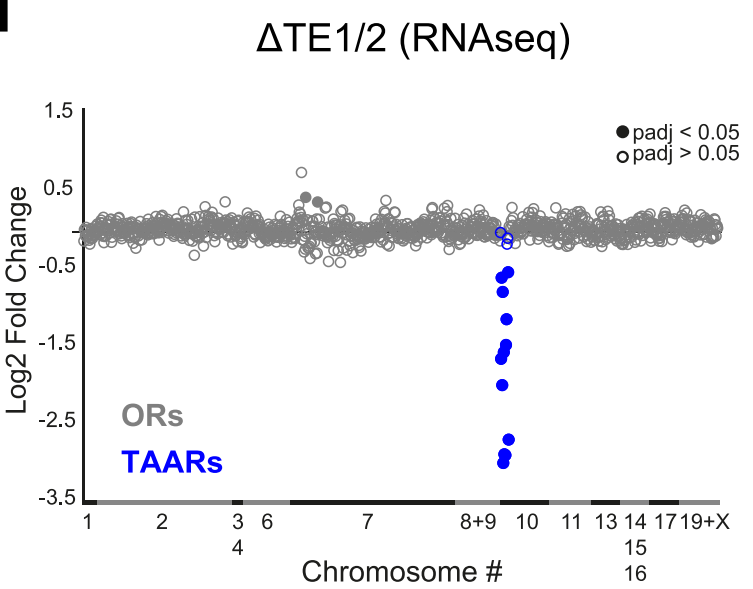

C

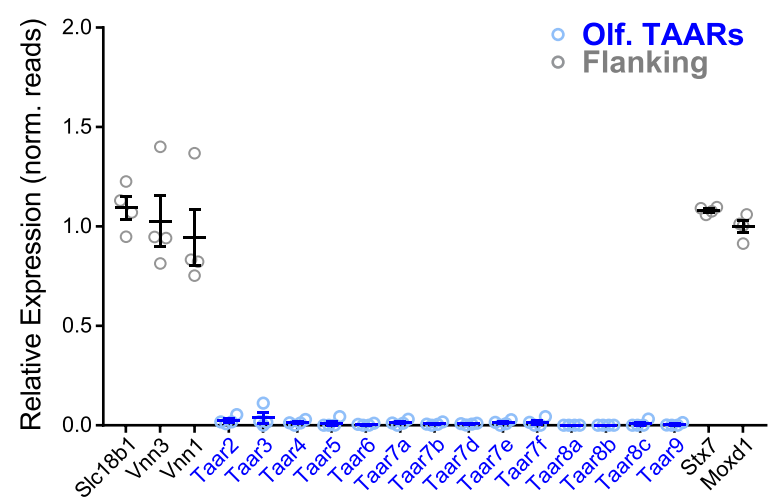

e

$\triangle T E 1 / 2$ (RNAseq)

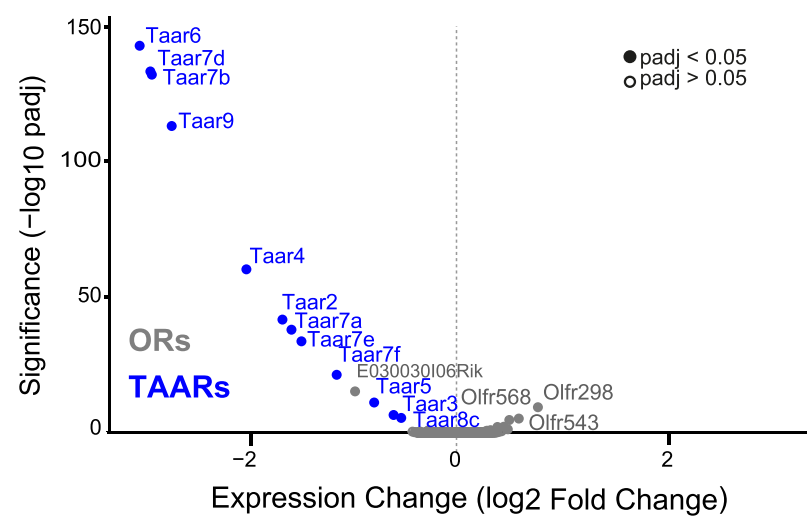

Fig. 6 TE1 and TE2 are required for TAAR gene expression. CRISPR-based gene editing was used to generate a double, cis deletion of both TE1 and TE2. a Diagram showing locations of gRNAs (gray) designed to target PAM sites flanking TE1 and TE2 (magenta). The double deletion ( $\Delta$ TE1/2) was generated on a chromosome harboring a previously targeted Taar4-RFP allele (red). Bottom, diagram of $\Delta T E 1 / 2$ in cis with Taar4-RFP. Deletion is marked (X). b Relative fold expression of TAAR (blue) and control OR (gray) genes measured using qPCR ( $2^{-\Delta \Delta C t}$ method) from olfactory mucosa. Error bars indicate mean \pm SEM. Taar8 primers amplify all 3 family members. $n=5$ mice per genotype. ${ }^{\star} p<0.0001$; two-sided, one-way ANOVA, Dunn-Šidák correction for multiple comparisons. c Relative expression ( $\Delta / w_{\text {avg }}$ normalized counts from DESeq2) for olfactory TAAR genes (blue) and genes flanking the TAAR cluster (gray). Error bars indicate mean \pm SEM. $n=4$ mice per genotype. d Change in expression ( $\log _{2}$ fold change) of all ORs (gray) and TAARs (blue) measured via RNAseq ( $n=4$ mice for each genotype). e Volcano plot ( $-\log _{10}$ adjusted $p$ value vs. $\log _{2}$ fold change) from DESeq 2 analysis of RNAseq data from homozygous $\Delta \mathrm{TE} 1 / 2$ and wild-type littermate control olfactory mucosa showing that TAAR genes are selectively affected by deletion. All genes with normalized counts $>10$ are plotted. Filled circles indicate genes with adjusted $p$ values $<0.05$ two-sided (DESeq2) Wald test corrected for multiple comparisons (Benjamini and Hochberg). $p$-values for RNAseq data can be found in Source Data File.

deletions does not clearly demarcate the functional extent of the enhancer sequence. In contrast, the deletion of TE1 and TE2 completely silences olfactory TAAR gene expression-clearly defining the functional boundaries of these enhancers.

Both T-elements and known OR enhancers comprise conserved HD binding sites, suggesting a common choice mechanism. However, previous studies have highlighted differences between TAAR and OR gene expression ${ }^{40,41}$. The TAARs appear to occupy a distinct nuclear compartment when compared to the $\mathrm{ORs}^{41}$. Moreover, ChIP-seq data assayed across the population of all mature OSNs show that the TAAR cluster lacks heterochromatic histone marks (H3K9Me3 and H4K20Me3) that 
a

T4-RFP

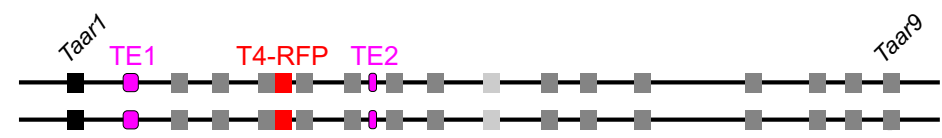

$\Delta$ TE $1 / 2$

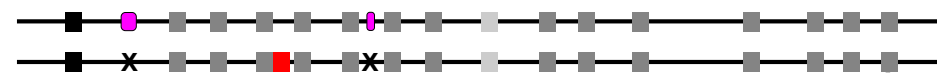

$\Delta \mathrm{TE} 1 / 2$

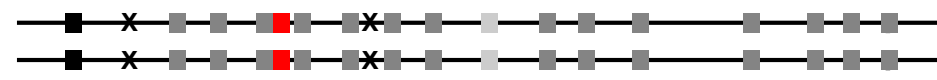

b
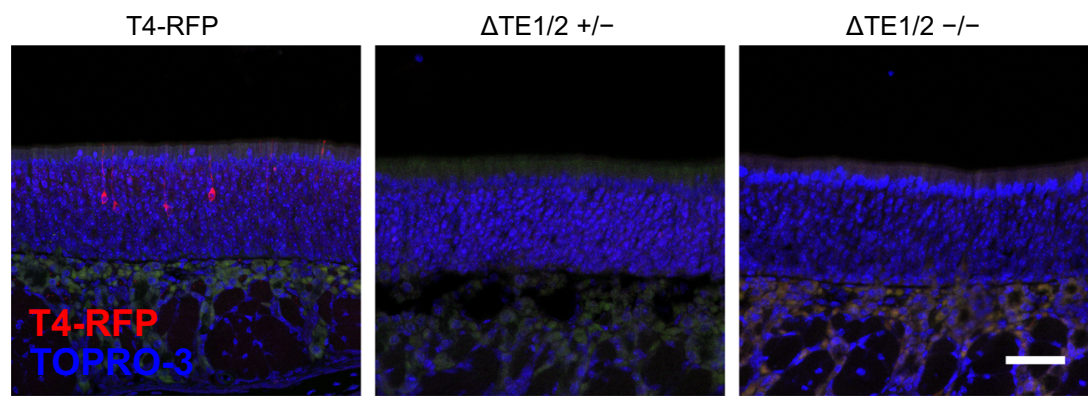

C

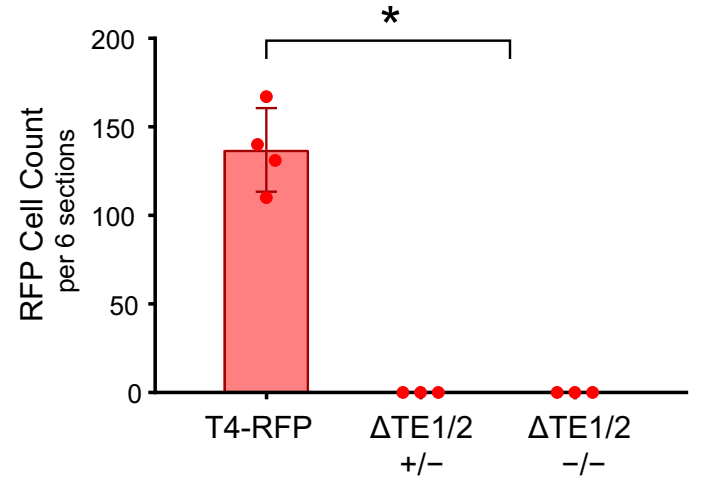

Fig. 7 TE1 and TE2 influence TAAR gene expression in cis not trans. a Three genotypes used to test for trans effects of TAAR elements. TE1 and TE2 are shown (magenta). Deletions are indicated (X). Taar4-RFP targeted allele (red) is linked to the double deletion, $\Delta T E 1 / 2$. b Confocal images of representative olfactory epithelium sections showing RFP-labeled OSNs (red) in Taar4-RFP mice (left). Nuclei are labeled with TOPRO-3 (blue). No labeled OSNs are seen in homozygous (right) or heterozygous (middle) $\Delta$ TE1/2 mice ( $n=3$ mice per genotype). c Average number of RFP+ OSNs counted in 6 representative olfactory epithelium sections from Taar4-RFP $(n=4), \Delta \operatorname{TE} 1 / 2$ heterozygous mice $(n=3)$, and $\Delta \operatorname{TE} 1 / 2$ homozygous mice $\left(n=3\right.$ mice). ${ }^{*} p<$ 0.0001 two-sided, ANOVA with contrasts. Error bars indicate mean \pm SEM. Scale bar $=50 \mu \mathrm{m}$.

are typically associated with class II OR clusters ${ }^{33,40}$. In addition, the available data indicate that the T-elements lack prominent binding of Lhx2, Ldb1, and Ebf1, which are features of some OR enhancers. One caveat to interpreting the ChIP-seq data is that TAAR-expressing OSNs represent a small fraction of the total number of mature OSNs. It is possible that repressive marks and transcription factor binding in the TAAR cluster might only be apparent in TAAR-expressing OSNs.

Despite these issues, the data indicate two things. First, in a majority of OSNs (most of which express class II ORs), the TAAR cluster is not marked in the same way as class II OR clusters. This suggests that the TAAR cluster may be silenced in OR-expressing OSNs independent of OR-typical heterochromatic marks. Second, in a majority of OSNs, the T-elements do not exhibit robust Lhx2 and Ldb1 binding to the same extent as many OR enhancers. This might suggest that the conserved HDs do not play a role in Telement function, or that the conserved HD sites bind factors other than Lhx2. Alternately, the T-element HD sites may be unable to associate with $\mathrm{Lhx} 2$ in most OSNs. One way to explain the ChIP-seq data is that the TAAR cluster may be inaccessible in
OR expressing neurons-something that could be accomplished by repression of T-element function (see below).

Previous work has shown that subpopulations of OSNs (either across zones, or within a zone) are restricted to express subsets of OR genes ${ }^{6,16,51-53}$. The mechanisms underlying cell-type-specific OR choice restrictions are not understood. One way to create a choice restriction would be to induce (or inhibit) expression of OR genes by activating (or repressing) cis-acting enhancers that coordinate expression in OR clusters. Such a mechanism was recently proposed for the class I OR cluster ${ }^{54}$. Likewise, one way to include or exclude TAAR expression from a population of OSNs might be to simultaneously control the function of TE1 and TE2. An intriguing possibility is that the SHiTE motif, which is common to both elements, could mediate such coordinated enhancer control. SHiTE might serve as a cell-type-specific activator of enhancer function. However, given that both TE1 and TE2 contain conserved HD sites, which are known to promote OR gene choice, it seems feasible that SHiTE might mediate repression of a default propensity for expression. We note that SHiTE contains a sequence AAAGTTT which is the reverse 
a

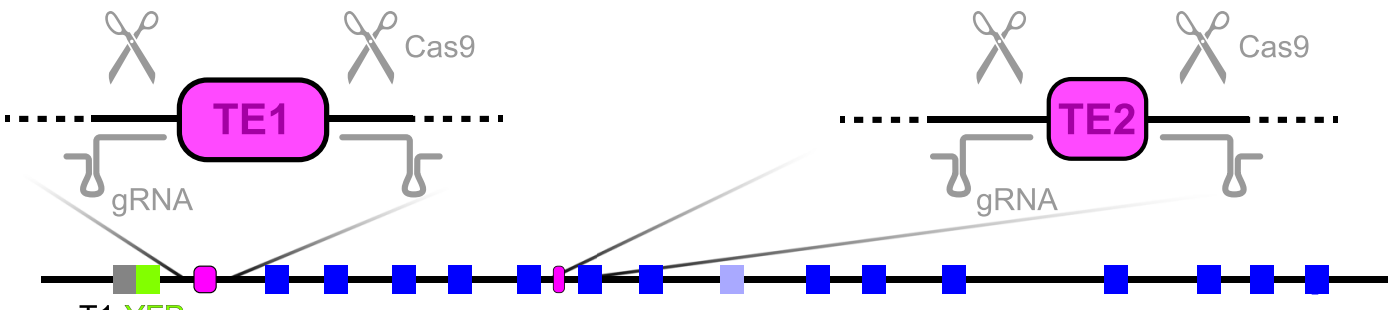

T1-YFP

4

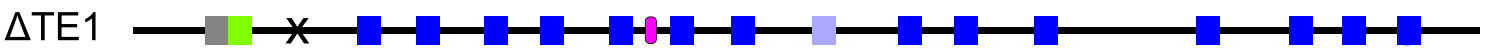

$\Delta$ TE2

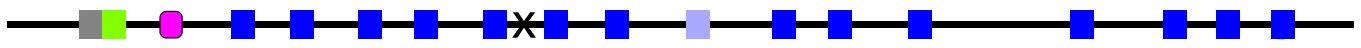

b

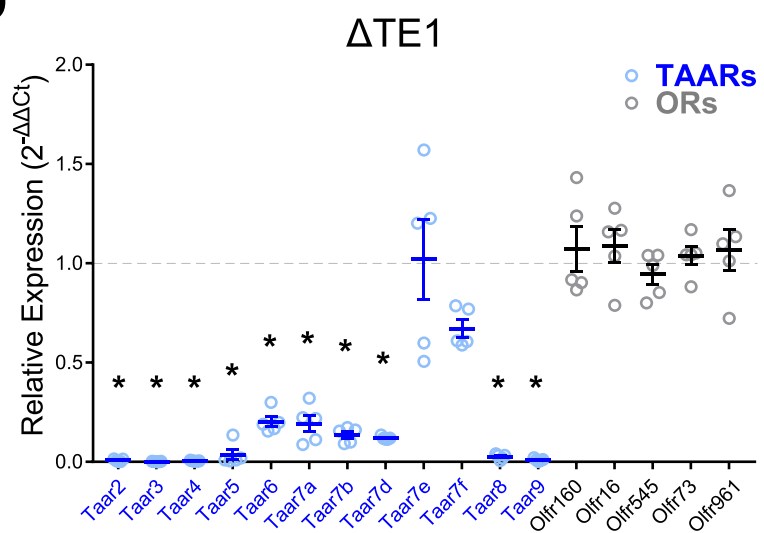

C

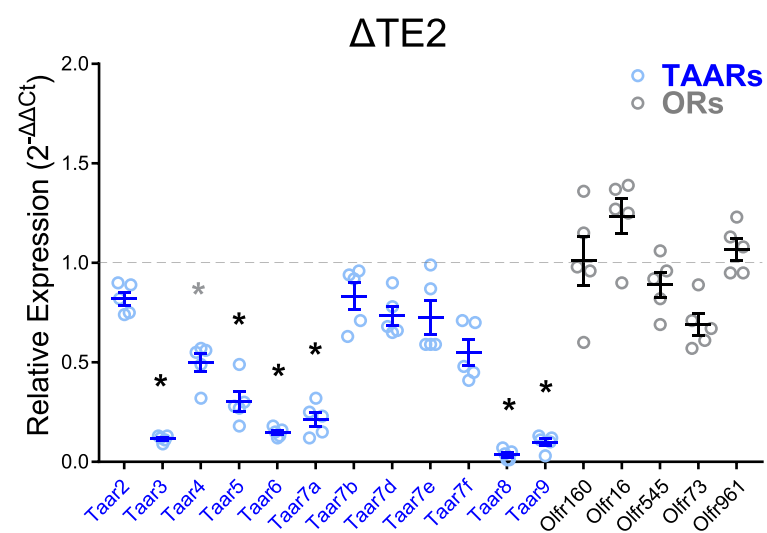

Fig. 8 TAAR enhancers function cooperatively. a Generation of single T-element deletions via CRISPR. Locations of gRNAs (gray) are shown relative to TE1 and TE2 (magenta). The individual $\triangle T E 1$ and $\triangle T E 2$ mutations were generated on a chromosome harboring a previously targeted Taar1-YFP allele (green). Bottom, diagram of $\Delta T E 1$ and $\triangle T E 2$ in cis with Taar1-YFP. Deletion is marked (X). b, c Relative fold expression of TAAR (blue) and control OR (gray) genes measured using GPCR (2- $\Delta \Delta \mathrm{Ct}$ method) from olfactory mucosa from $\Delta \mathrm{TE1}$ and $\Delta \mathrm{TE} 2$ mice. Error bars indicate mean \pm SEM. Taar8 primers amplify all 3 family members. $n=5$ mice per genotype. ${ }^{\star} p<0.01$; one-way ANOVA, Dunn-Šidák correction for multiple comparisons.

complement of AAACTTT, which is conserved in the class I OR enhancer $\mathrm{J}^{20}$ and that is part of an extended HD motif ${ }^{30}$. While the contribution of the class I motif to gene choice is unclear ${ }^{55}$, there may be an as yet uncovered common function.

Our discovery of cooperative, cis-acting enhancers in the TAAR cluster that share common features with OR enhancers suggests that TAAR and OR gene choice proceed via a fundamentally similar mechanism. Thus, identifying which characteristics of gene choice are common between TAARs and ORs will help elucidate mechanisms that are essential for olfactory singular expression.

\section{Methods}

All experimental procedures were approved by the Northwestern University Institutional Animal Care and Use Committee.

Mouse strains. The T4-RFP (Taar4-IRES-tauCherry) and $\triangle \mathrm{T} 4$-RFP (GAP-Cher$\mathrm{ry} \rightarrow$ Taar4) strains were described previously ${ }^{6}$.

$\triangle T 4$-YFP transgenes. A $129 \mathrm{~S} 7 \mathrm{BAC}$ clone ${ }^{56}$ (bMQ-215O19) containing the Taar4 locus was modified via recombineering by the insertion of an AscI flanked galK cassette $3 \mathrm{nt}$ downstream of the Taar4 coding sequence. A $7.9 \mathrm{~kb}$ fragment of the modified BAC (corresponding to GRCm39/mm39 chr10: 23,830,822-23,838,778) was isolated by gap repair, and the coding sequence replaced with that of Venus $\mathrm{YFP}^{57}$ preceded by a Kozak consensus (GCCACCATG). This base transgene (YFP $\rightarrow$ T4tg) contains $\sim 2.3 \mathrm{~kb}$ upstream of the Taar4 transcription start site and $1.3 \mathrm{~kb}$ downstream of the coding sequence (including the endogenous polyadenylation site), and was flanked with PmeI sites for linearization. Enhancers (5x21HD, TE1 and TE2) were amplified by PCR and inserted into a XhoI site that is $\sim 2 \mathrm{~kb}$ upstream of the transcriptional start. $5 \mathrm{xHD}-\triangle \mathrm{T} 4 \mathrm{YFPtg}$ contains a 5 copy repeat of the extended $21 \mathrm{bp}$ homeodomain sequence (ACA-

TAACTTTTTAATGAGTCT), as previously described ${ }^{30}$. TE1- $\Delta$ T4YFPtg and TE2$\Delta$ T4YFPtg contain one copy of TE1 (corresponding to GRCm39/mm39 chr10: $23,806,280-23,806,978$ ) or TE2 (chr10: $23,863,682-23,864,027)$, respectively. Transgenes were linearized with PmeI purified, and injected into C57BL/6 J zygotes to generate transgenic founders.

$\triangle T E 1, \triangle T E 2, \triangle T E 1 / 2$ CRISPR alleles. PAM sequences on the $5^{\prime}$ and $3^{\prime}$ ends of each putative enhancer element were targeted with two guide RNAs each. The recognition sequences were cloned into pX458 (Addgene \#48138) and the gRNAs transcribed in vitro using T7 RNA polymerase. The gRNAs and wild-type Cas9 RNA were injected into mouse zygotes that were compound heterozygous for two mutations: AU1-Taar1-IRES-tauVenus and Taar4-IRES-tauCherry. Founders were screened by PCR and direct sequencing. Mutant alleles with the following deletions were selected: GRCm39/mm39 chr10: 23,806,234-23,807,226 (993 bp) for $\Delta \mathrm{TE1}$, and chr10: $23,863,677-23,864,656(980 \mathrm{bp})$ for $\Delta \mathrm{TE} 2$, each isolated in $c i s$ with the AU1-Taar1-IRES-tauVenus mutation. An allele with deletions of both elements in tandem $(\Delta$ TE1/2) was also selected: chr10: 23,806,230-23,807,225 (996 bp) for $\Delta$ TE1 and chr10: 23,863,677-23,864,655 (979 bp) for $\Delta$ TE2, and was isolated in cis with the Taar4-IRES-tauCherry mutation. All of the alleles were backcrossed for six generations onto a C57BL/6 J background, then intercrossed. For all mutations, homozygous mice appear healthy and show normal fertility. Mice were housed in a specific-pathogen free barrier vivarium on a $14 / 10$ light/dark cycle at $21-24^{\circ} \mathrm{C}$ and $50 \%$ relative humidity with access to food and water ad libitum.

Histology. Postnatal day 30 mice were anesthetized and transcardially perfused with $4 \%$ paraformaldehyde. The nose was dissected and post-fixed at $4{ }^{\circ} \mathrm{C}$ 
overnight, decalcified in $0.5 \mathrm{M}$ EDTA overnight, and cryoprotected in $15 \%$ sucrose for $1 \mathrm{~h}$ and $30 \%$ sucrose overnight (both at $4^{\circ} \mathrm{C}$ ). OCT-embedded samples were cryosectioned at $12 \mu \mathrm{m}$. Combined in situ hybridization/immunohistochemistry in transgenic lines ${ }^{16,58}$ was performed using digoxigenin-labeled riboprobes and antidigoxigenin-alkaline phosphatase Fab fragments at 1:1000 dilution (Roche, 11093274910). Proteinase K (10 $\mu \mathrm{g} / \mathrm{ml}$; Takara 740396$)$ treatment time was adjusted to allow for immunostaining. YFP protein was detected with a chicken anti-GFP antibody (Abcam, ab13970) at 1:500, coupled with a rabbit anti-chicken peroxidase secondary (Invitrogen, 61-3120) 1:500. Tyramide signal amplification was performed using TSA amplification reagent (Akoya Biosciences) at $1 / 10$ of the prescribed biotinyl-tyramide concentration ${ }^{58}$. Probes for ORs/TAARs were transcribed using T7 or SP6 RNA polymerase from cloned and sequenced partial or full-length coding sequence templates that were amplified from olfactory epithelium cDNA or genomic DNA. Template plasmids were linearized and purified by phenol:chloroform extraction. The probe pools were- class I: olfr623, olfr 578 , olfr691, olfr672, olfr668, and olfr545; class II: olfr749, olfr745, olfr771, olfr1031, olfr62, and olfr247; TAAR: taar2, taar4, taar5, taar6, taar7a, and taar9.

All fluorescence images were acquired using a Zeiss LSM880 laser scanning confocal microscope using Zen 2.3 SP1 software (Carl Zeiss Microscopy). For whole-mount analysis of olfactory bulbs and epithelia, genetically encoded fluorescent markers were visualized in unfixed samples. Wholemount images were collected as tile-scanned z-stacks and displayed as flattened projections.

\section{Gene expression analysis}

$q P C R$. Olfactory mucosa was isolated from P30 TAAR element deletion mice and matching wild-type littermate controls. RNA was purified using the Qiagen RNeasy Kit (\#74134) with on-column DNase treatment using Qiagen RNase-free DNase (\#EN0521). Oligo-dT primed cDNA was synthesized using SuperScript III Reverse transcriptase (\#18080044). qPCR was performed according to MiQE guidelines ${ }^{59}$, using iQ SYBR Green (\#1708884). $\Delta$ Ct values were calculated using the geometric mean of the housekeeping genes Bgus, Gnal, and Ncam. Primers were tested for efficiency (95-105\%). Primer sequences are found in Supplementary Table 1.

RNAseq. Olfactory mucosa was isolated from postnatal day (P30) TAAR element deletion mice and matching wild-type littermate controls. RNA was purified using the Qiagen RNeasy Kit (\#74134) and on-column DNase treatment was performed using Qiagen RNase-free DNase (\#EN0521). Stranded, OligodT selected cDNA libraries were synthesized, multiplexed, and run on an Illumina HiSeq4000 (PE100) by the University of Chicago Genomics Facility. Reads were aligned to a modified GRCm38.p6 mouse genome containing extended TAAR exon and UTR information using STAR aligner v2.4.260. Differential expression analysis was performed using DeSeq2 v1.30.1 ${ }^{61}$ using default settings.

Statistics and reproducibility. qPCR data were analyzed by one-way ANOVA corrected for multiple comparisons (Dunn-Šidák) on the raw delta Ct values to test the null hypothesis of equal Ct values between samples. Differential expression in RNAseq data was estimated using the Wald test correcting for multiple comparisons using the method of Benjamini and Hochberg. All tests were done using Prism v7 (GraphPad) and R v1.2 (www.r-project.org).

Gene expression patterns in transgenic mice were documented in at least 3-6 animals for each independent line. RNAseq and in situ hybridization experiments were performed once using the number of independent samples (animals) indicated in the main text.

Reporting summary. Further information on research design is available in the Nature Research Reporting Summary linked to this article.

\section{Data availability}

RNAseq datasets generated and analyzed during the currents study are available via the Geo database (accession \#GSE171241). Previously published datasets ${ }^{21,27}$ that were analyzed during the current study are also available (accession \#GSE93570 for ATACseq, Lhx2 ChIP-seq, Ebf ChIP-seq and GSE112152 for Ldb1 ChIP-seq). Eutherian conservation track analyzed in the current study can be found on the UCSC Genome Browser. Source data are provided with this paper.

Received: 10 September 2020; Accepted: 18 May 2021;

Published online: 18 June 2021

\section{References}

1. Reinius, B. \& Sandberg, R. Random monoallelic expression of autosomal genes: stochastic transcription and allele-level regulation. Nat. Rev. Genet. 16, 653-664 (2015).
2. Eckersley-Maslin, M. A. \& Spector, D. L. Random monoallelic expression: regulating gene expression one allele at a time. Trends Genet. 30, 237-244 (2014).

3. Gendrel, A. V. et al. Developmental dynamics and disease potential of random monoallelic gene expression. Dev. Cell 28, 366-380 (2014).

4. Serizawa, S., Miyamichi, K. \& Sakano, H. One neuron-one receptor rule in the mouse olfactory system. Trends Genet. 20, 648-653 (2004).

5. Chess, A., Simon, I., Cedar, H. \& Axel, R. Allelic inactivation regulates olfactory receptor gene expression. Cell 78, 823-834 (1994).

6. Pacifico, R., Dewan, A., Cawley, D., Guo, C. \& Bozza, T. An olfactory subsystem that mediates high-sensitivity detection of volatile amines. Cell Rep. 2, 76-88 (2012)

7. Zhang, X. \& Firestein, S. The olfactory receptor gene superfamily of the mouse. Nat. Neurosci. 5, 124-133 (2002).

8. Nei, M., Niimura, Y. \& Nozawa, M. The evolution of animal chemosensory receptor gene repertoires: roles of chance and necessity. Nat. Rev. Genet. 9, 951 (2008).

9. Niimura, Y., Matsui, A. \& Touhara, K. Corrigendum: extreme expansion of the olfactory receptor gene repertoire in African elephants and evolutionary dynamics of orthologous gene groups in 13 placental mammals. Genome Res. 25, 926 (2015).

10. Freitag, J., Ludwig, G., Andreini, I., Rossler, P. \& Breer, H. Olfactory receptors in aquatic and terrestrial vertebrates. J. Comp. Physiol. A 183, 635-650 (1998).

11. Godfrey, P. A., Malnic, B. \& Buck, L. B. The mouse olfactory receptor gene family. Proc. Natl Acad. Sci. USA 101, 2156-2161 (2004).

12. Qasba, P. \& Reed, R. R. Tissue and zonal-specific expression of an olfactory receptor transgene. J. Neurosci. 18, 227-236 (1998).

13. Vassalli, A., Rothman, A., Feinstein, P., Zapotocky, M. \& Mombaerts, P. Minigenes impart odorant receptor-specific axon guidance in the olfactory bulb. Neuron 35, 681-696 (2002).

14. Serizawa, S. et al. Negative feedback regulation ensures the one receptor-one olfactory neuron rule in mouse. Science 302, 2088-2094 (2003).

15. Fuss, S. H., Omura, M. \& Mombaerts, P. Local and cis effects of the $\mathrm{H}$ element on expression of odorant receptor genes in mouse. Cell 130, 373-384 (2007).

16. Bozza, T. et al. Mapping of class I and class II odorant receptors to glomerular domains by two distinct types of olfactory sensory neurons in the mouse. Neuron 61, 220-233 (2009).

17. Vassalli, A., Feinstein, P. \& Mombaerts, P. Homeodomain binding motifs modulate the probability of odorant receptor gene choice in transgenic mice. Mol. Cell Neurosci. 46, 381-396 (2011).

18. Khan, M., Vaes, E. \& Mombaerts, P. Regulation of the probability of mouse odorant receptor gene choice. Cell 147, 907-921 (2011).

19. Markenscoff-Papadimitriou, E. et al. Enhancer interaction networks as a means for singular olfactory receptor expression. Cell 159, 543-557 (2014).

20. Iwata, T. et al. A long-range cis-regulatory element for class I odorant receptor genes. Nat. Commun. 8, 885 (2017).

21. Monahan, K., et al. Cooperative interactions enable singular olfactory receptor expression in mouse olfactory neurons. Elife 6, e28620 (2017).

22. Cichy, A., Shah, A., Dewan, A., Kaye, S. \& Bozza, T. Genetic depletion of Class I odorant receptors impacts perception of carboxylic acids. Curr. Biol. 29, 2687-2697 (2019).

23. Wang, M. M., Tsai, R. Y., Schrader, K. A. \& Reed, R. R. Genes encoding components of the olfactory signal transduction cascade contain a DNA binding site that may direct neuronal expression. Mol. Cell Biol. 13, 5805-5813 (1993)

24. Kudrycki, K., Stein-Izsak, C., Behn, C., Grillo, M., Akeson, R. \& Margolis, F. L. Olf-1-binding site: characterization of an olfactory neuron-specific promoter motif. Mol. Cell Biol. 13, 3002-3014 (1993).

25. Zhang, Y. Q., Breer, H. \& Strotmann, J. Promotor elements governing the clustered expression pattern of odorant receptor genes. Mol. Cell Neurosci. 36, 95-107 (2007).

26. Hoppe, R., Weimer, M., Beck, A., Breer, H. \& Strotmann, J. Sequence analyses of the olfactory receptor gene cluster mOR37 on mouse chromosome 4 . Genomics 66, 284-295 (2000).

27. Monahan, K., Horta, A. \& Lomvardas, S. LHX2- and LDB1-mediated trans interactions regulate olfactory receptor choice. Nature 565, 448-453 (2019).

28. Hirota, J. \& Mombaerts, P. The LIM-homeodomain protein Lhx2 is required for complete development of mouse olfactory sensory neurons. Proc. Natl Acad. Sci. USA 101, 8751-8755 (2004).

29. Zhang, G., Titlow, W. B., Biecker, S. M., Stromberg, A. J. \& McClintock, T. S. Lhx2 determines odorant receptor expression frequency in mature olfactory sensory neurons. eNeuro 3, ENEURO.0230-0216.2016 (2016).

30. D'Hulst, C. et al. MouSensor: a versatile genetic platform to create super sniffer mice for studying human odor coding. Cell Rep. 16, 1115-1125 (2016).

31. Nishizumi, H., Kumasaka, K., Inoue, N., Nakashima, A. \& Sakano, H. Deletion of the core-H region in mice abolishes the expression of three proximal odorant receptor genes in cis. Proc. Natl Acad. Sci. USA 104, 20067-20072 (2007). 
32. Dewan, A. et al. Single olfactory receptors set odor detection thresholds. Nat. Commun. 9, 2887 (2018).

33. Magklara, A. et al. An epigenetic signature for monoallelic olfactory receptor expression. Cell 145, 555-570 (2011).

34. Lyons, D. B. et al. Heterochromatin-mediated gene silencing facilitates the diversification of olfactory neurons. Cell Rep. 9, 884-892 (2014).

35. Lomvardas, S., Barnea, G., Pisapia, D. J., Mendelsohn, M., Kirkland, J. \& Axel, R. Interchromosomal interactions and olfactory receptor choice. Cell 126, 403-413 (2006).

36. Clowney, E. J. et al. Nuclear aggregation of olfactory receptor genes governs their monogenic expression. Cell 151, 724-737 (2012).

37. Lindemann, L., Ebeling, M., Kratochwil, N. A., Bunzow, J. R., Grandy, D. K. \& Hoener, M. C. Trace amine-associated receptors form structurally and functionally distinct subfamilies of novel $\mathrm{G}$ protein-coupled receptors. Genomics 85, 372-385 (2005).

38. Xie, Z. \& Miller, G. M. Trace amine-associated receptor 1 as a monoaminergic modulator in brain. Biochem. Pharm. 78, 1095-1104 (2009).

39. Liberles, S. D. \& Buck, L. B. A second class of chemosensory receptors in the olfactory epithelium. Nature 442, 645-650 (2006).

40. Johnson, M. A. et al. Neurons expressing trace amine-associated receptors project to discrete glomeruli and constitute an olfactory subsystem. Proc. Natl Acad. Sci. USA 109, 13410-13415 (2012).

41. Yoon, K. H. et al. Olfactory receptor genes expressed in distinct lineages are sequestered in different nuclear compartments. Proc. Natl Acad. Sci. USA 112, E2403-E2409 (2015).

42. Schwartz, S. et al. PipMaker-a web server for aligning two genomic DNA sequences. Genome Res. 10, 577-586 (2000).

43. Wang, M. M. \& Reed, R. R. Molecular cloning of the olfactory neuronal transcription factor Olf-1 by genetic selection in yeast. Nature 364, 121-126 (1993).

44. Michaloski, J. S., Galante, P. A. \& Malnic, B. Identification of potential regulatory motifs in odorant receptor genes by analysis of promoter sequences. Genome Res. 16, 1091-1098 (2006).

45. Travis, A., Hagman, J., Hwang, L. \& Grosschedl, R. Purification of early-B-cell factor and characterization of its DNA-binding specificity. Mol. Cell Biol. 13, 3392-3400 (1993).

46. Grant, C. E., Bailey, T. L. \& Noble, W. S. FIMO: scanning for occurrences of a given motif. Bioinformatics 27, 1017-1018 (2011).

47. Feinstein, P., Bozza, T., Rodriguez, I., Vassalli, A. \& Mombaerts, P. Axon guidance of mouse olfactory sensory neurons by odorant receptors and the beta2 adrenergic receptor. Cell 117, 833-846 (2004).

48. Lewcock, J. W. \& Reed, R. R. A feedback mechanism regulates monoallelic odorant receptor expression. Proc. Natl Acad. Sci. USA 101, 1069-1074 (2004).

49. Zapiec, B. \& Mombaerts, P. The zonal organization of odorant receptor gene choice in the main olfactory epithelium of the mouse. Cell Rep. 30, 4220-4234 e4225 (2020).

50. Fei A., et al. Coordination of two enhancers drives expression of olfactory trace amine-associated receptors. Nat. Commun. https://doi.org/10.1038/ s41467-021-23823-4 (2021).

51. Ressler, K. J., Sullivan, S. L. \& Buck, L. B. A zonal organization of odorant receptor gene expression in the olfactory epithelium. Cell 73, 597-609 (1993).

52. Vassar, R., Ngai, J. \& Axel, R. Spatial segregation of odorant receptor expression in the mammalian olfactory epithelium. Cell 74, 309-318 (1993).

53. Miyamichi, K., Serizawa, S., Kimura, H. M. \& Sakano, H. Continuous and overlapping expression domains of odorant receptor genes in the olfactory epithelium determine the dorsal/ventral positioning of glomeruli in the olfactory bulb. J. Neurosci. 25, 3586-3592 (2005).

54. Enomoto, T. et al. Bcl11b controls odorant receptor class choice in mice. Commun. Biol. 2, 296 (2019).

55. Iwata, T., Tomeoka, S. \& Hirota, J. A class I odorant receptor enhancer shares a functional motif with class II enhancers. Sci. Rep. 11, 510 (2021).

56. Adams, D. J. et al. A genome-wide, end-sequenced 129Sv BAC library resource for targeting vector construction. Genomics 86, 753-758 (2005).

57. Nagai, T., Ibata, K., Park, E. S., Kubota, M., Mikoshiba, K. \& Miyawaki, A. A variant of yellow fluorescent protein with fast and efficient maturation for cellbiological applications. Nat. Biotechnol. 20, 87-90 (2002).
58. Ishii, T., Omura, M. \& Mombaerts, P. Protocols for two- and three-color fluorescent RNA in situ hybridization of the main and accessory olfactory epithelia in mouse. J. Neurocytol. 33, 657-669 (2004).

59. Bustin, S. A. et al. The MIQE guidelines: minimum information for publication of quantitative real-time PCR experiments. Clin. Chem. 55, 611-622 (2009).

60. Dobin, A. et al. STAR: ultrafast universal RNA-seq aligner. Bioinformatics 29, 15-21 (2013).

61. Love, M. I., Huber, W. \& Anders, S. Moderated estimation of fold change and dispersion for RNA-seq data with DESeq2. Genome Biol. 15, 550 (2014).

\section{Acknowledgements}

This work was supported by grants from NIH/NIDCD to A.S (F32DC015962) and T.B. (R01DC013576), and from Northwestern University to M.R. (Office for Undergraduate Research). P.F is supported by R21GM126304 and A.R. is supported by the Research Initiative for Scientific Enhancement (RISE) program at Hunter College, funded by NIH grant GM060665. We thank Sarah Kaye, Hardik Patel, and Amanda Menzie for technical support, Bill Kath for advice on bioinformatics, Lynn Doglio and the Transgenesis and Targeted Mutagenesis Laboratory at Northwestern University for production of transgenic mice, and Pieter Faber and the Genomics Facility at University of Chicago for highthroughput sequencing. We are indebted to Tom Meade and Hao Li in the Department of Chemistry at Northwestern for synthesis of HNPP.

\section{Author contributions}

T.B and P.F. conceived the project and designed experiments. T.B. generated genetically modified mouse strains. A.S. performed RNAseq and differential gene expression analysis. M.R. performed in situ hybridization and histological cell counts. M.R., A.R, and P.F. performed bioinformatics analyses. A.S, M.R., and T.B. wrote the manuscript with input from all authors.

\section{Competing interests}

The authors declare no competing interests.

\section{Additional information}

Supplementary information The online version contains supplementary material available at https://doi.org/10.1038/s41467-021-23824-3.

Correspondence and requests for materials should be addressed to T.B

Peer review information Nature Communications thanks the anonymous reviewers for their contribution to the peer review of this work.

Reprints and permission information is available at http://www.nature.com/reprints

Publisher's note Springer Nature remains neutral with regard to jurisdictional claims in published maps and institutional affiliations.

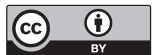

Open Access This article is licensed under a Creative Commons Attribution 4.0 International License, which permits use, sharing, adaptation, distribution and reproduction in any medium or format, as long as you give appropriate credit to the original author(s) and the source, provide a link to the Creative Commons license, and indicate if changes were made. The images or other third party material in this article are included in the article's Creative Commons license, unless indicated otherwise in a credit line to the material. If material is not included in the article's Creative Commons license and your intended use is not permitted by statutory regulation or exceeds the permitted use, you will need to obtain permission directly from the copyright holder. To view a copy of this license, visit http://creativecommons.org/ licenses/by/4.0/.

(C) The Author(s) 2021 